\title{
PLANS FOR THE IMPROVEMENT AND EXTENSION OF THE FK4
}

\author{
(Invited Paper)
}

WALTER FRICKE

Astronomisches Rechen-Institut, Heidelberg, F.R. Germany

\begin{abstract}
Recent absolute and differential observations of fundamental stars have indicated the need for a revision of the fundamental reference system represented by the positions and proper motions of the FK4. The revision of the FK4 will be based on modern fundamental and differential observations of high accuracy which allow (a) the extension of the FK4 to fainter stars, (b) the improvement of the system, (c) the derivation of individual corrections to positions and proper motions, and (d) the determination of the equinox and its motion. The completion of the programme, resulting in the FK5 and a Supplement giving data in the system of FK 5 for bright stars and faint objects (including optical counterparts of radio sources), may be expected about 1980 .
\end{abstract}

\section{Principles of Establishing a Fundamental Frame of Reference}

The Fourth Fundamental Catalogue (FK4) represents the conventional fundamental frame of reference adopted by the IAU in 1961 (IAU Trans. 10, 79 and 11B, 1967). This catalogue gives the positions and proper motions of 1535 stars that are fairly uniformly distributed over the sky and are brighter than visual magnitude 7.5. For practical reasons the system is that of the Earth's equator as the fundamental plane from which declinations are reckoned, and the vernal equinox as the origin for reckoning right ascensions. The system is established by absolute observations of positions of stars and members of the planetary system at various epochs.

Any approach to improve the fundamental frame of reference has to begin with a critical survey of the possibilities for a revision of the principles underlying the construction of the reference frame. The choice of the equatorial system is loaded with a number of difficulties due to the orbital motion of the Earth and to the motion of the equator and equinox. It is therefore appropriate to ask whether any other choice could be made. Well-known are the advantages of an extragalactic reference frame represented by positions of galaxies in a net-work over the sky. The net of positions can be established, in principle, without relation to the equatorial system, and a zero-point can be arbitrarily chosen. In practice, however, we are still far away from a realization of this possibility, at least, as far as the independence of the net of extragalactic positions from the equatorial system is concerned. In an unforeseen way the new radio-interferometer techniques which provide declinations and right ascensions of radio sources without reference to previously determined positions, support the continued use of the equatorial system as the proper choice for the near future.

There are other important arguments in favour of no change in the principles of establishing a fundamental reference frame at present, but in favour of a drastic improvement of the conventional system on the basis of available observational ma- 
terial by means of classical methods and by all possible attempts at relating the system of bright stars to the radio astrometric system. First, I may mention that the FK4 can hardly serve its purposes satisfactorily for more than about 20 years from the date of its completion. With advancing epoch of observation, the accuracy of star places computed from the FK4 decreases, mainly due to the errors of the proper motions. Second, the increase in the accuracy of modern observations and an increasing diversity of applications demand for more reliable ephemerides. Third, during the past two decades a large number of absolute and differential observations of FK4 stars and fainter stars have been made with improved techniques with the classical instruments, the meridian circle, vertical circle, and Danjon's astrolabe, such that it is justified to start with the improvement of the FK4 and of extending it, hopefully, to the ninth visual magnitude in a new fundamental catalogue, the FK5.

The work before us consists of several steps, most of which are closely related to each other and must be done one after the other, and some are independent from each other and can be done simultaneously. Even with a perfect organization of the work, the completion of the FK5 can hardly be expected before 1980. The various steps may be described by the following main headings:

(1) Documentation of basic observational data and study of the techniques that have been applied;

(2) Extension of the FK4 system to a fainter magnitude limit;

(3) Derivation of systematic corrections to the FK4 from absolute observations;

(4) Derivation of individual corrections to the star places and proper motions in the FK4 from absolute and differential observations;

(5) Improvement of precessional values and elimination of errors due to incorrect motion of the equinox (a change in the conventional values of precession requires international agreement);

(6) Establishment of a Supplement to the FK5 giving accurate data of bright stars and faint objects including optical counterparts of radio sources.

I will proceed now in describing various aspects of these tasks in more detail except item (5) concerning precession which will be one of the general themes of the Joint Discussion No. 1 at the XV IAU General Assembly.

\section{Documentation of Observational Data}

The documentation of observations relevant to the improvement and extension of the FK4 includes the punching of the data of a great number of observational catalogues; general catalogues which are the result of a compilation of different sources will not be used. We are dealing with two groups of catalogues, the first consists of those since about 1900 , in which the observers have included stars fainter than visual magnitude 7.5, and where measures have been taken to avoid errors depending on the magnitude. These observations will contribute to the extension of the FK4 to fainter stars. The second group consists of catalogues which have become available since the completion of the FK4. They may contribute to the improvement of the 
systematic and individual accuracy, and, of course, also to the extension of the FK4. The total number of catalogues which will have to be prepared in a form suitable for data processing will be between 160 and 200. Many of these have already been punched in a joint project of the U.S. Naval Observatory and the Yale Observatory and others at Heidelberg. In addition, some observatories have provided their catalogues on punched cards, but these are favourable exceptions to a rule which in future, should be followed. Together with the documentation of observations goes the study of the methods, which have been applied by the observers, in order to decide for which purposes the material can be used. In fact, it is often not easy to find out to what extent observations are absolute, quasi-absolute, or differential to a system which can not be expected to be the system of the FK4.

We expect that work at the documentation will take us another few years, and that even after its completion we should be prepared to include in the program data that may become available within the next three years from now on. This implied a continuous contact between the observers and the compilers of the FK5 at Heidelberg.

\section{Extension of the Fundamental System to Fainter Stars}

At the time of the compilation of the FK4 the available observations were not sufficient for an extension of the system beyond the visual magnitude 7.5 without considerable loss in systematic and individual accuracy. Almost all observations made before 1900 are seriously affected by errors depending on the magnitude, and even after 1900 it has taken some time to convince observers that provision for the elimination of 'magnitude equations' in declinations and right ascensions is necessary. During the past two decades the situation has considerably improved. Furthermore, the international reference star programs AGK3R (reference stars for the AGK3) and SRS (southern reference stars) have provided observations down to about ninth magnitude which are either strictly in the system of the FK4 or in instrumental systems whose relations to the FK4 are well determined. There are about 40000 stars distributed over the whole sky in the reference star programs. The observations in the AGK3R were shared by ten observatories and completed in 1963. The resulting accuracy of $\pm 0.005 \sec \delta$ and \pm 0 . 116 in right ascension and declination is excellent. One may therefore consider the AGK3R as the most comprehensive sample of stars in the northern sky suitable for the selection of stars down to ninth magnitude for the extension of the fundamental system. At Heidelberg, we have adopted the AGK3R as the 'master catalogue' from which stars in the magnitude interval from 7.5 to about 9 will be selected for inclusion in the FK5. In the southern sky, the SRS catalogue shall serve the same purpose. The selection will depend on the observational history of the stars, and the final choice will have to be made on the basis of the accuracy of proper motions that can be derived from all suitable observations made from about 1900 onwards. Concerning the spectral composition of a fundamental catalogue, I am of the opinion that the distribution should not be restricted to a narrow range in spectral classes. How useful it would be, if at least a few stars brighter 
than ninth magnitude with radio emission could be included in the FK5! The advantages of such stars for linking the radio astrometric system to the fundamental system have only recently become apparent.

Besides the primary criterion of accuracy of positions and proper motions, secondary criteria may be the distribution of the stars in the sky and in distance from the Sun. For practical reasons, the representatives of a fundamental reference frame should be distributed fairly uniformly in the sky. On the other hand it is attractive to include in the efforts of deriving proper motions of highest systematic precision as many stars as possible at large distances from the Sun. This aim implies that MK spectral types and accurate photometric data are available.

Besides the AGK3R and SRS catalogue as sources for the extension of the fundamental system, the FK4 Sup catalogue will provide additional material. FK4 Sup stars have been included in many observational programs, and many of them will fulfil conditions of sufficient systematic and individual accuracy for inclusion in the FK5. Although it is not possible to make a well-founded estimate of the number of stars in the FK 5 before the investigation of the observational history of the stars in the AGK3 R, the SRS catalogue and the FK4 Sup will be completed, I expect that the number of 1535 stars in FK 4 will have to be increased to at least 3000 and at most to 5000 in the FK5.

\section{Improvement of the System and of the Accuracy of Individual Data in the FK4}

Concerning the prospects of an improvement of the system of the FK4 and the derivation of individual corrections I refer to the report to be given by Dr Gliese except for a few questions of general nature. These questions concern (1) large scale inhomogeneities in the system over the sky, which have become apparent by systematic deviations of modern positions from the FK4 in various regions of the sky, and (2) errors in the determination of the equinox and the equator point. Among the inhomogeneities appear to be most prominent the errors in right ascensions in the zone $-50^{\circ}$ to $-80^{\circ}$. They were found by the joint effort of Chilean and Russian astronomers working at different instruments at the Santiago Observatory, and they were confirmed by other observers as well. If one takes into account that the FK 4 system south of $-30^{\circ}$ rests mainly on observations of a single observatory, the Cape observatory, such errors are not surprising. It is more surprising that no serious errors in the southern declination system have become known. The absolute observations made during the SRS program are, however, not yet completed, and the final derivation of corrections to the FK4 system in the south must wait for the results which may become available within the next few years.

The equator point and the equinox are being determined by observations of the Sun and other bodies of the planetary system. In fact, almost the same series of observations contribute to the determination of both, and they also yield corrections to the obliquity of the ecliptic. The results depend mainly on the absolute measurement of declinations. Such measurements determine more easily the position of a great circle 
in the sky, the equator, than the position of the Sun in the equator and in the solstices. From the results which we have derived for the position of the equator in the FK4these results have not shown any secular change - I do not expect that more recent observations will give us an appreciable correction. The situation is different with the determination of the equinox and the obliquity. The position of the equinox in the FK4 is defined by $N_{1}+\Delta N_{1}$, where $N_{1}$ denotes the equinox in the right ascension system of equatorial stars in the Catalogue of 1098 Standard Clock and Zodiacal Stars by Newcomb (1898). In the same year this equinox was applied by Newcomb in the Catalogue of Fundamental Stars. Later, appreciable corrections $\Delta N_{1}$ were found from the observations of the Sun and other bodies of the planetary system. All observations carried out from about 1900 to 1950 have provided values of $\Delta N_{1}$ in the interval

$$
-0.028 \geqslant\left(\Delta N_{1}\right)_{\text {Cat }} \geqslant-0.073
$$

with no indication of a significant secular change. In the FK4 the correction $\Delta N_{1}$ $=-0.050$ was adopted but it has not been taken into account in the proper motions. In fact, all compilers of fundamental catalogues after Newcomb have avoided to eliminate this error in the hope that it could be dealt with in connection with a revision of Newcomb's value of general precession. I consider the elimination of a fictitious non-precessional motion of the equinox as a necessity, and the FK5 should be freed from this error independently of a decision on a change in the precessional values. The system of positions and proper motions in right ascension in the FK5 will rest on observations after 1900 only. It would therefore be illogical, if the zero-point determination would contain a relic from the 19th century that has been recognized as a systematic error due to changes in the techniques of observation.

A similar situation exists with the determination of the obliquity $\varepsilon$ of the ecliptic. Individual corrections $\Delta \varepsilon$ to Newcomb's obliquity from observations of the Sun and planets show a pronounced secular change of the order of

$$
\Delta \dot{\varepsilon}=-0 \text {.'3 per century, }
$$

if observations before 1900 are taken into accoùnt. This change cannot be explained by planetary precession. The suspicion that we are also here dealing with systematic errors of old observations is supported by the fact that the observations after 1900 do not yield a significant secular change. If there were a real change, I would agree with Aoki (1968) that the equator of the Earth is performing a rotation about an axis passing through the vernal equinox and that the effect would need a physical explanation.

In summarizing this part of my talk, it can be said that the improvement of the equator point and the equinox require an extensive investigation of all relevant observations of the Sun and other bodies of the planetary system and of the consequences of changes for the system of the FK5. Fortunately, this investigation can be made independently from the study of systematic differences between the FK4 and modern observations which will contribute to the elimination of regional errors in the fundamental system. 
The derivation of individual corrections to the data in the FK4 will rest upon all observations that have become available from about 1950 onwards and that have been carried out by absolute and differential techniques. The problem of determining weights to the observations will have to be reconsidered in view of the large material which is already incorporated in the FK4. In the past, there has often been some suspicion that new observations, which have been added in the formation of a new fundamental catalogue, have not received the proper weight for updating the fundamental system. Every care has to be taken that suspicion of this sort is unfounded.

\section{Compilation of a Supplement to the FK5}

Among the observational material now available there will be data for stars which cannot be included in the new system without loss of accuracy. For such stars the computer methods applied to the fundamental stars may, however, yield positions and proper motions which are much better than those which can be derived from photographic plates taken at several epochs. It appears attractive to include such stars in a Supplement to the FK5 whenever it is possible to give their positions at mean epoch with an accuracy appreciably better than $\pm 0.01 \sec \delta$ and \pm 0 ". 1 (standard errors in right ascension and declination, respectively) and their proper motions with an accuracy better than \pm 0 ". 40 per century in each coordinate. This aim has been reached for the stars in the FK3 Sup, and these have turned out to be exceedingly useful for many purposes. We are therefore planning to compile a Supplement Catalogue to the FK5 resulting partly from a by-product of the work at the FK5. To another part this Supplement may incorporate entirely new material, namely, the positions of faint objects in the system of the FK5.

Among the most attractive faint objects that may be taken into account are the optical counterparts of compact extragalactic radio sources. They are suited for relating the fundamental system to the radio astrometric system of extragalactic sources. Moreover, stars of our galactic system which are radio sources are of interest. For both classes of objects optical positions can be obtained by photographic means with an accuracy of about $\pm 0.015 \sec \delta$ and \pm 0 ". 15 (standard errors in right ascension and declination, respectively). Although this accuracy is inferior to that of radio astrometric measurements, and although the positions of optical counterparts may not be strictly identical with those of the radio sources, the optical astronomers should not be discouraged from proceeding with the photographic measurements. The Supplement to the FK5 may contribute to the encouragement of measurements in the future, and with this purpose in mind, it should be considered as a challenging sample of stars for future work.

Finally, reference shall be made to some papers giving information on results of observations and problems connected with the improvement and extension of FK4. Desiderata for a new fundamental catalogue have been described by Fricke and Gliese (1968), who have also reported on new observations. More recent observations have been discussed by Gliese $(1970,1974)$. From the reports by Anguita et al. $(1968,1971$, 
1974) and Høg and Nikoloff (1974) it has become clear that the FK4 system in right ascension requires an appreciable correction in the declination zone $-50^{\circ}$ to $-80^{\circ}$. Zverev (1968) has reported on absolute observations made in the USSR that indicate some significant regional deviations from the FK4 in right ascension, and Yasuda (1972) has reported on semi-absolute observations which resulted in corrections to right ascensions. Fricke $(1970,1973)$ has considered the question of whether the fundamental system can be replaced by a reference system of galaxies, and has described the prospects for an extension of the fundamental system to faint objects and radio sources. The present situation as to fundamental systems of positions and proper motions has been reviewed by Fricke (1972).

\section{References}

Anguita, C., Carrasco, G., Loyola, P., Shishkina, V. N., and Zverev, M. S. : 1968, in L. Perek (ed.), Highlights of Astronomy, D. Reidel Publ. Co., Dordrecht-Holland, p. 292.

Anguita, C., Zverev, M. S., Carrasco, G., and Polozhentsev, D. D.: 1971, Izv. Glav. Astron. Obs. Pulkovo, No. $189-190,83$.

Anguita, C., Carrasco, G., Loyola, P., Naumova, A. A., Nemiro, A. A., Polozhentsev, D. D., Shishkina, V. N., Taibo, R., Timashkova, G. M., Varin, M. P., Varina, V. A., and Zverev, M. S. : 1974, this volume p. 35 .

Fricke, W.: 1970, in W. J. Luyten (ed.), 'Proper Motions', IAU Colloq. 7, 105.

Fricke, W.: 1972, Ann. Rev. Astron. Astrophys. 10, 101.

Fricke, W.: 1973, Mem. Soc. Astron. Italiana 43, 751.

Fricke, W. and Gliese, W.: 1968, in L. Perek (ed.), Highlights of Astronomy, D. Reidel Publ. Co., DordrechtHolland, p. 301.

Gliese, W.: 1970, in W. J. Luyten (ed.), 'Proper Motions', IAU Colloq. 7, 146.

Gliese, W.: 1974, this volume p. 31.

Høg, E. and Nikoloff, I.: 1974, this volume, p. 79.

Yasuda, H.: 1972, Publ. Astron. Soc. Japan 24, 247.

Zverev, M. S. : 1968, in L. Perek (ed.), Highlights of Astronomy, D. Reidel Publ. Co., Dordrecht-Holland, p. 286.

\section{DISCUSSION}

Vasilevskis: If discrepancies between the Lick and Pulkovo proper motions are explained, are you going to make use of proper motions measured with reference to galaxies?

Fricke: We will not use them in the FK5, since there we need to know positions at various epochs which we can compare with the positions provided by observations made with different instruments. The fundamental proper motions will be derived from the positions. We may, however, consider the possibility of taking them into account in the Supplement to the FK5, if positions become known from other sources.

Murray: I don't see why you ignore the information on the motion of the equinox given by the stellar kinematics, since you have to use a kinematic hypothesis to determine the precession.

Fricke: In deriving a fundamental catalogue from observations alone, we have to determine the equinox from absolute observations of the Sun and other bodies of the planetary system, and if the determination yields an unexplained motion of the equinox then we shall be in a difficult position. Boss was faced with that difficulty in 1906, and he proposed to eliminate the unexplained motion of the equinox by making use of the information given by stellar kinematics, because he considered the planetary observations to be of poor quality. At present, we have clear indications that the observations of the Sun and planets from 1900 onwards do not show a secular variation of the equinox. We will investigate these observations in detail, and 
I hope they will be good enough to eliminate the motion of the equinox without recourse to stellar kinematics.

Strand: In view of the proposal to provide a Supplement to the FK 5 containing positions of radio sources, emphasis should be placed on the need to observe these optically, perhaps by co-operation between several observatories. Such co-operation will probably be necessary since few observatories have instrumentation to cover the entire range from the brighter stars to the optically faint objects associated with the radio sources.

Fricke: I agree entirely with Dr Strand's remark.

Wood: I would be inclined to wager that it would be a quotation from Clemence that coordinates should be defined to conform to an operational method of determining them. This seems to make it difficult to get away from the equator as a fundamental plane.

Fricke: I agree with Clemence's opinion.

Gubbay: Does Prof. Fricke wish to include positions of compact radio sources or only their optical counterparts in the FK5? If the radio positions per se are to be included, how should we develop an ethos for radio position measurement acceptable to the Catalogue? Should we have a table of primary reference sources? Furthermore the ephemeral quality of some compact components and the dependence of their 'visibility' on the orientation of the baseline may introduce difficulties in relating different observations over a period of time.

Fricke: Radio position measurements per se should be included as well as that of their optical counterparts. We look to the radio astronomers to help resolve the problems of cataloguing radio position measurements.

Klock: In regard to Prof. Fricke's concern on the improvement of the equator point with solar observations made in the last twenty years, it is noted that a serious problem exists, inasmuch that the number of transit circles making solar observations has dwindled to an alarmingly small number today. 